\title{
A Regularization Scheme for the AdS/CFT Correspondence
}

\author{
W. Mück* and K. S. Viswanathan ${ }^{\dagger}$ \\ Department of Physics, Simon Fraser University, Burnaby, British Columbia, V5A1S6 Canada
}

July 2, 2021

\begin{abstract}
The prescription of the AdS/CFT correspondence is refined by using a regularization procedure, which makes it possible to calculate the divergent local terms in the CFT two-point function. We present the procedure for the example of the scalar field.
\end{abstract}

*Email address: wmueck@sfu.ca

${ }^{\dagger}$ Email address: kviswana@sfu.ca 
It has been stated in most papers on this subject that the correspondence between a fields theory on anti-de Sitter space (AdS) and a conformal field theory (CFT) on its boundary is formally described by the formula [1, 2]

$$
\mathrm{e}^{-I_{\text {AdS }}[\phi]}=\left\langle\exp \int d^{d} x \phi_{0}(x) \mathcal{O}(x)\right\rangle,
$$

where the action $I_{A d S}$ is calculated on-shell for a field configuration $\phi$ satisfying a Dirichlet condition on the AdS boundary and the boundary value $\phi_{0}$ couples as a current to the conformal field $\mathcal{O}$ living on the AdS boundary. Thus, the formula (四) enables one to calculate correlation functions of the field $\mathcal{O}$ in the boundary conformal field theory. There are two subtle points, which this formal description does not address. First, a Dirichlet boundary value problem in general is not well defined for anti-de Sitter space, since a generic field does not propagate to the boundary. This point has been addressed by formulating the theory with a boundary lying inside the anti-de Sitter space. Using this approach the two-, three- and four-point functions of various fields have been calculated (see [3] for a recent comprehensive list of references). Secondly, although the $\epsilon$-prescription yields the non-contact contributions to the correlators in agreement with conformal field theory, the singular contributions for coincidence points have so far escaped a direct calculation. Previous work has focused on obtaining a finite action by interpreting the correlators as distributions [4] and regularizing the action by adding boundary counter terms [5, 6]. Two loop corrections to Super Yang Mills correlators have been calculated in [7] and contribute only to the contact terms. However, we think that a regularization scheme must include a prescription on how to calculate the divergent contact contributions before any counterterms are added.

The aim of this letter is to provide a refined prescription of the AdS/CFT correspondence, with which non-local and local terms of the CFT two-point function can be calculated. We would like to emphasize that it is not our aim to obtain regularized CFT correlation functions.

We shall use the conventional representation of anti-de Sitter space by the space $x^{i} \in \mathbb{R}$, $(i=1, \ldots d), x^{0}>0$ with the metric

$$
d s^{2}=\left(x^{0}\right)^{-2} d x^{\mu} d x^{\mu} .
$$

Let us consider the example of a scalar field [8], which satisfies the equation of motion

$$
\left(\nabla^{2}-m^{2}\right) \phi(x)=\left[x_{0}^{2} \partial_{\mu} \partial_{\mu}-x_{0}(d-1) \partial_{0}-m^{2}\right] \phi(x)=0 .
$$

A solution of equation (3) can be written in the form

$$
\phi(x)=\int d^{d} y K(x, \mathbf{y}) \phi_{\epsilon}(\mathbf{y})
$$

where $\phi_{\epsilon}(\mathbf{y})$ is some boundary field and the bulk-boundary kernel is given by

$$
K(x, \mathbf{y})=\int \frac{d^{d} k}{(2 \pi)^{d}}\left(\frac{x_{0}}{\epsilon}\right)^{\frac{d}{2}} \frac{K_{\alpha}\left(k x_{0}\right)}{K_{\alpha}(k \epsilon)} \mathrm{e}^{-i \mathbf{k} \cdot(\mathbf{x}-\mathbf{y})-\mu k^{2}} .
$$

Here, $K_{\alpha}$ is a modified Bessel function (Mac Donald function) and $\alpha$ is related to the mass parameter by

$$
\alpha=\sqrt{\frac{d^{2}}{4}+m^{2}}
$$


In addition, we have introduced the regulating factor $\mathrm{e}^{-\mu k^{2}}$ in order to make the integral well defined for all values of $x_{0}$ and $\mathbf{x}-\mathbf{y}$. In the limit $\mu \rightarrow 0$ equation (4) reduces to the standard Dirichlet kernel. It will turn out that the limits $\mu \rightarrow 0$ and $\epsilon \rightarrow 0$ should be taken simultaneously with $\mu$ being of order $\epsilon^{2}$.

The CFT two-point function is determined by the boundary normal derivative of the kernel (4), which is given by

$$
\left.\partial_{0} K(x, \mathbf{y})\right|_{\epsilon}=\frac{1}{\epsilon} \int \frac{d^{d} k}{(2 \pi)^{d}}\left\{\frac{d}{2}-\alpha+k \frac{\partial}{\partial k} \ln \left[(k \epsilon)^{\alpha} K_{\alpha}(k \epsilon)\right]\right\} \mathrm{e}^{-i \mathbf{k} \cdot(\mathbf{x}-\mathbf{y})-\mu k^{2}} .
$$

Consider first the case of non-coincident points $\mathbf{x}$ and $\mathbf{y}$. As without regularization, we use the expansion

$$
z^{\alpha} K_{\alpha}(z)=2^{\alpha-1} \Gamma(\alpha)\left[1+\sum_{j=1}^{\infty} \frac{1}{j !(1-\alpha)_{j}}\left(\frac{z}{2}\right)^{2 j}-\frac{\Gamma(1-\alpha)}{\Gamma(1+\alpha)}\left(\frac{z}{2}\right)^{2 \alpha} \sum_{j=0}^{\infty} \frac{1}{j !(1+\alpha)_{j}}\left(\frac{z}{2}\right)^{2 j}\right],
$$

where we used the notation $(a)_{j}=\Gamma(a+j) / \Gamma(a)$. Proceeding to expand the logarithm in equation (5) one obtains

$$
z \frac{\partial}{\partial z} \ln \left[z^{\alpha} K_{\alpha}(z)\right]=\frac{z^{2}}{2(1-\alpha)}+\cdots-\frac{\Gamma(1-\alpha)}{\Gamma(1+\alpha)} 2^{1-2 \alpha} \alpha z^{2 \alpha}+\cdots,
$$

where the first set of dots indicates analytic terms of order $z^{2 n}(n>1)$ and the second set higher order non-analytic terms. Substituting equation (7) into equation (5) we recognize integrals of the type

$$
\begin{aligned}
\int \frac{d^{d} k}{(2 \pi)^{d}} k^{\beta} \mathrm{e}^{-i \mathbf{k} \cdot \mathbf{x}-\mu k^{2}} & =\frac{|\mathbf{x}|^{1-\frac{d}{2}}}{(2 \pi)^{\frac{d}{2}}} \int_{0}^{\infty} d k k^{\frac{d}{2}+\beta} J_{\frac{d}{2}-1}(k|\mathbf{x}|) \mathrm{e}^{-\mu k^{2}} \\
& =\frac{\Gamma\left(\frac{d+\beta}{2}\right)}{2^{d} \pi^{\frac{d}{2}} \Gamma\left(\frac{d}{2}\right) \mu^{\frac{d+\beta}{2}}} \Phi\left(\frac{d+\beta}{2} ; \frac{d}{2} ;-\frac{|\mathbf{x}|^{2}}{4 \mu}\right)
\end{aligned}
$$

Here, $\Phi(a, c, z)$ is the degenerate hypergeometric function. For $\mathbf{x} \neq 0$ we can take the $\mu \rightarrow 0$ limit and replace $\Phi$ with the leading term of its asymptotic expansion [9], which yields

$$
\int \frac{d^{d} k}{(2 \pi)^{d}} k^{\beta} \mathrm{e}^{-i \mathbf{k} \cdot \mathbf{x}-\mu k^{2}} \stackrel{\mu \rightarrow 0}{=} \frac{2^{\beta} \Gamma\left(\frac{d+\beta}{2}\right)}{\pi^{\frac{d}{2}} \Gamma\left(-\frac{\beta}{2}\right)} \frac{1}{|\mathbf{x}|^{d+\beta}} .
$$

We notice that for the analytic terms, $\beta=2 n$, the gamma function in the denominator diverges, so the integral becomes zero. The same holds for the subleading terms of the asymptotic expansion. Hence, the analytic terms do not contribute to the finite distance two-point function. On the other hand, for the leading non-analytic term, $\beta=2 \alpha$, we obtain (still for $\mathbf{x} \neq \mathbf{y}$ )

$$
\left.\partial_{0} K(x, \mathbf{y})\right|_{\epsilon} \stackrel{\mu \rightarrow 0}{=} 2 \alpha c_{\alpha} \frac{\epsilon^{2 \alpha-1}}{|\mathbf{x}-\mathbf{y}|^{2 \Delta}}
$$


where $\Delta=d / 2+\alpha$ and $c_{\alpha}=\Gamma(\Delta) /\left(\pi^{d / 2} \Gamma(\alpha)\right)$. The higher order non-analytic terms in equation (17) contribute with higher powers of $\epsilon$ and can be neglected in the $\epsilon \rightarrow 0$ limit. Hence, we find agreement with previous results [8]. A nice and simple check of this procedure is provided by the conformally coupled scalar field, $\alpha=1 / 2$, where the calculation can be done exactly.

Let us now turn to the local terms in the two-point function. In this case, it is more useful not to carry out the expansion (7), since all its terms would contribute because of $\Phi(a, c, 0)=1$. Consider instead the expression (5) directly with $\mathbf{x}=\mathbf{y}$. Using the identity

$$
z \frac{\partial}{\partial z} \ln \left[z^{\alpha} K_{\alpha}(z)\right]=-z \frac{K_{\alpha-1}(z)}{K_{\alpha}(z)}
$$

and changing the integration variable to $\mathbf{s}=\sqrt{\mu} \mathbf{k}$ we find

$$
\left.\partial_{0} K(x, \mathbf{x})\right|_{\epsilon}=\frac{1}{\epsilon(4 \pi \mu)^{\frac{d}{2}}}\left[\frac{d}{2}-\alpha-\frac{2 a}{\Gamma\left(\frac{d}{2}\right)} \int d s s^{d} \mathrm{e}^{-s^{2}} \frac{K_{\alpha-1}(a s)}{K_{\alpha}(a s)}\right],
$$

where the parameter $a$ denotes the ratio $a=\epsilon / \sqrt{\mu}$. The $s$-integral is well-defined for all positive $a$, although in general not elementary. However, we know that it represents some positive number. Hence, we obtain

$$
\left.\partial_{0} K(x, \mathbf{x})\right|_{\epsilon}=-\frac{\gamma}{\epsilon(4 \pi \mu)^{\frac{d}{2}}}
$$

where $\gamma$ is a regularization dependent parameter satisfying

$$
\alpha-\frac{d}{2}<\gamma<\infty
$$

Moreover, if $2 \alpha<d$, i.e. for $-d^{2}<4 m^{2}<0$, one could determine $a$ such that $\gamma=0$. For example, in the conformally coupled case, $\alpha=1 / 2$, one finds

$$
a_{\frac{1}{2}}=\frac{(d-1) \Gamma\left(\frac{d}{2}\right)}{2 \Gamma\left(\frac{d+1}{2}\right)} .
$$

The contribution (12) to the two-point function can be compensated by adding a local counterterm

$$
I_{c}=-\frac{\gamma}{2} \int d^{d} x \epsilon^{-d}\left[\phi_{\epsilon}(\mathbf{x})\right]^{2}
$$

to the AdS action of a scalar field. However, one must notice that this only eliminates the contact term, but does not regularize the two-point function.

In conclusion, we have presented in this letter a refined prescription of the AdS/CFT correspondence, which invokes a regularization scheme in order to calculate the contact contributions to the CFT correlators. Our calculation shows that in general there is such a contribution to the two-point function, but it can be compensated by adding a covariant local surface term to the AdS action. Alternatively, it might serve as a regulator for the CFT two-point function and thus eliminate the need to add counterterms. We think that this is an interesting topic for further study.

This work was supported in part by a grant from NSERC. W. M. is grateful to Simon Fraser University for financial support. 


\section{References}

[1] S. S. Gubser, I. R. Klebanov and A. M. Polyakov, Phys. Lett. B428, 105 (1998), hep-th/9802109

[2] E. Witten, Adv. Theor. Math. Phys. 2, 253 (1998), hep-th/9802150

[3] E. D'Hoker, D. Z. Freedman, S. D. Mathur, A. Matusis and L. Rastelli, Graviton exchange and complete 4-point functions in the AdS/CFT correspondence, hep-th/9903196

[4] I. Ya. Aref'eva and I. V. Volovich, Phys. Lett. B433, 49 (1998), hep-th/9804182

[5] G. Chalmers and K. Schalm, Holographic Normal Ordering and Multi-particle States in the AdS/CFT Correspondence, hep-th/9901144

[6] R. Emparan, C. V. Johnson and R. C. Myers, Surface Terms as Counterterms in the AdS/CFT Correspondence, hep-th/9903238

[7] F. Gonzalez-Rey, P. Kulik and I. Y. Park, Non-renormalization of two and three Point Correlators of $N=4$ SYM in $N=1$ Superspace, hep-th/9903094

[8] W. Mück and K. S. Viswanathan, Phys. Rev. D 58, 041901 (1998), hep-th/9804035

[9] J. B. Seaborn, Hypergeometric functions and their applications, Springer Verlag (1991) 